\title{
A Novel Image De-blur Algorithm based on Wavelet Packet Transform Model and Framework
}

\author{
Xinxin Xie $^{\mathrm{a}}$ and Wenzhun Huang ${ }^{\mathrm{b}^{*}}$ \\ School of Information Engineering, Xijing University, Xi'an 710123, China \\ a346148500@qq.com, bhuangwenzhun@xijing.edu.cn \\ *The corresponding Author
}

\begin{abstract}
Keywords: Image De-blur; Wavelet packet; Transform model; Systematic design; Modelling
\end{abstract}
\begin{abstract}
In this paper, we propose the novel image de-blur algorithm based on the wavelet packet transform model and the framework. Wavelet analysis has the characteristics of the multi-resolution, multi-scale, when it is applied to basic image enhancement, wavelet coefficient on different scales in different nonlinear transformation function, the different frequency of the image detail is enhanced, the effect is better than traditional enhancement algorithm. However, due to the wavelet enhancement changes to the brightness of image is not large, the images of the uneven illumination or inadequate, the traditional wavelet enhancement effect. Therefore, we propose wavelet packet transform model for optimization. The effectiveness and feasibility are verified.
\end{abstract}

\section{Introduction}

The purpose of general image enhancement is to improve the image visual effect, for a given image applications, purposefully stressed global or local characteristics of the image, expand the differences between different object in the image features to meet the needs of the specific analysis. In the process of image enhancement, not this paper analyzes the reasons of images is qualitative, not necessarily close to the original image after processing. Image enhancement technology based on the enhanced processing in space is different, divided into airspace based algorithm and based on frequency domain algorithm two kinds big. Based on the algorithm of the airspace to handle directly do arithmetic of the image grayscale, based on the algorithm of frequency domain is in a transform domain of the image to some correction, image transform coefficient value is a kind of indirect enhancement algorithm.

According to the literature review, the general features of the blurred images could be summarized as follows. (1) Image in part caused by a lack of access to light and is located in the shaded area in grey value is low, and the background blend together, while other parts with the high contrast, but image dynamic range is big to make this part of the information in the original image are extracted. (2) Due to the poor acquisition images when ambient light conditions or to the collect images the equipment itself, have on the overall image grey value is low, low image contrast, interested in area it is difficult to identify, such as the infrared image and to obtain images of the evening. (3) Image in metal smooth surface or curved surfaces, makes the collected images appear highlights phenomenon. Highlights the phenomenon not only makes the image information is difficult to extract and changed the face of the original image that lead to further processing more difficult [1-3].

Image enhancement technology does not consider images with qualitative reason, attenuation of unwanted image information. Traditionally, in order to achieve a satisfactory result, for a given image enhancement goal need to apply a variety of the complementary image enhancement technology. (1) Image smoothing often make image blurred boundary, outline and its real because of the fuzzy image by the average or integral operation. In order to reduce the influence of this kind of adverse effects, and often to reverse it make the image clear. Using the image sharpening technology, strengthen the image of the target boundary and image details. (2) Grayscale histogram reflects the image grayscale and appears this kind of gray level of the relationship between the probabilities of graphics. A uniform quantization of natural image gray histogram is usually in low frequency on gray area is larger, the image is darker, details of the area of the often can't see clearly. To make the image clear, the natural idea is to make the 
image gray scale is large dynamic range, and small grayscale and frequency after changing its frequency becomes larger, the transformation of image gray histogram into equilibrium within the large dynamic range, this is the histogram modification technology. (3) Wavelet analysis theory and method of research for many engineering provides a new tool and method of it in the time domain and frequency domain at the same time has good localization property that can be focused to signal any details. The characteristics of the wavelet analysis, particularly suitable for the image the non-stationary signal analysis and processing that could be reflected in figure one.
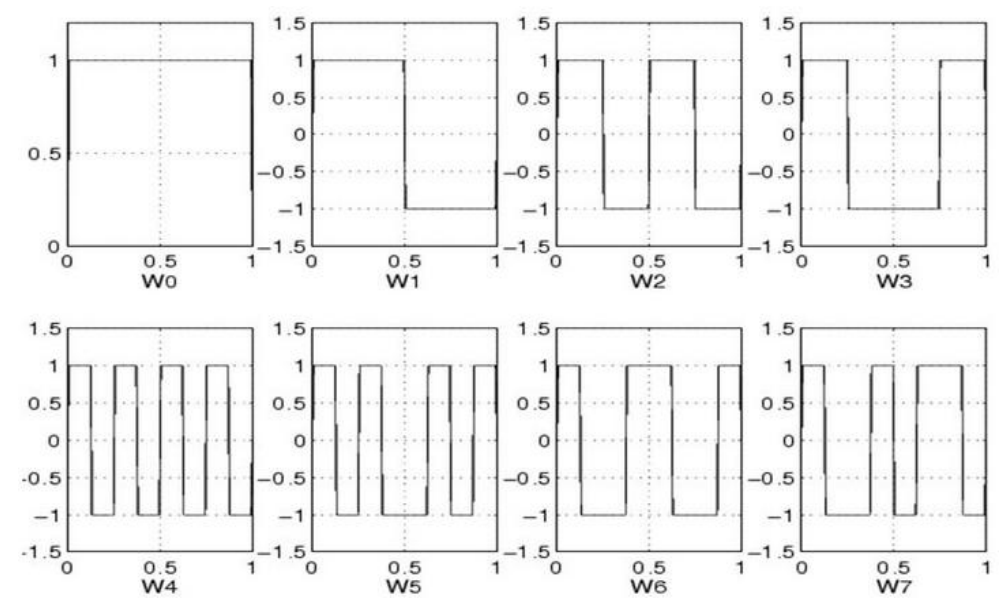

Figure 1. The Wavelet Packet Transformation Function Pattern

In this article, we propose the novel image de-blur algorithm based on the wavelet packet transform model and the framework. Image restoration is to remove the noise in the image as is a very important part of image processing. The process of image restoration is to the true nature of the image and also namely from the degraded image restoration to can really reflect the landscape image. In sub-sections, we will propose our novel perspectives and methodologies.

\section{Systematic Description of the Algorithm}

The Survey of the Image Enhancement Methods. Digital image enhancement technique, to smooth the image after histogram specification method is adopted to improve the enhancement and Laplace sharpening methods, histogram specification in prominent edge profile at the same time, not to any residual noise in image enhancement, this kind of method for the contrast is not high as details of the blurred image is very appropriate. Against the disadvantage of global histogram method, various local histogram equalization methods are put forward. According to the equilibrium degree of overlapping sub-block, local histogram equalization algorithm can be divided into the overlapping sub-block don't overlap, sub-block and blocks overlap [4-5].

Local method only considers the grayscale distribution in a window without taking into account the overall characteristics of the image, so easy to weaken the image layers. In the recent years, the improved method based on histogram equalization method was put forward, such as the overlap of the equalization algorithm is proposed on the basis of the image pair according to the luminance average block recursive decomposition, get a series of different gray scale range of sub images, then for each child image in the corresponding sub-block gray within the scope of application of overlap algorithm of histogram equalization, and finally merge the equilibrium result of sub image.

$$
\left\{\begin{array}{l}
I(x, y)=L(x, y) * R(x, y) \\
L(x, y)=I(x, y) * G(x, y)
\end{array}\right.
$$

Most local enhancement method the compressed image information dynamic range and enhancing the dark, but not enough light and shadow part of the original image enhancement original uniform illumination parts in the form of uniform gradient distribution, while the gradient range is small, light and 
shadow parts can be compressed at the same time expand the bright part of the other parts of the gradient or the shaded part of the image gradient, the compressed image dynamic range and enhancing local contrast and this part makes information easier to identify. Using frequency division filter the image is divided into two parts of basic low frequency and high frequency, only for low frequency component of the histogram equalization, then, it will be treated with the low frequency part and high frequency part of a merger, again to the combined information, median filtering to remove noise and get the final enhanced images, not only keep the overall contrast of image and the detail information, and in addition to the problem of noise amplification with blurring pattern.

The Wavelet Packet Transform Model. Wavelet packet analysis is a kind of basic signal analysis method can provide more detailed results. It will signal frequency band multi-level classification and subdivision of multiresolution wavelet analysis without high frequency part of further decomposition, according to the analysis of features of the signal, the adaptively select band, make it match the signal spectrum, thus raised the time-frequency resolution [6].

Wavelet function and wavelet packet function is one of the biggest difference is that: in the process of orthogonal wavelet decomposition, is generally low frequency coefficient decomposition into a new low frequency coefficient and high frequency coefficient vector, and then will continue to a new low frequency coefficient vector is decomposed into two parts, and decomposition of high frequency coefficients are no longer. In the wavelet packet decomposition, each high frequency as coefficient vector also like low frequency part, continue to be broken down into two parts. Thus, wavelet packet than wavelet decomposition provides a more elaborate analysis results.

$$
\left\{\begin{array}{c}
u_{2 n}(t)=\sqrt{2} \sum h(k) u_{n}(2 t-k) \\
u_{2 n+1}(t)=\sqrt{2} \sum g(k) u_{n}(2 t-k)
\end{array}\right.
$$

Above the wavelet packet decomposition is specific to one dimensional function, for a 2D image, with expansion coefficient of the fast algorithm is similar to one dimensional case.

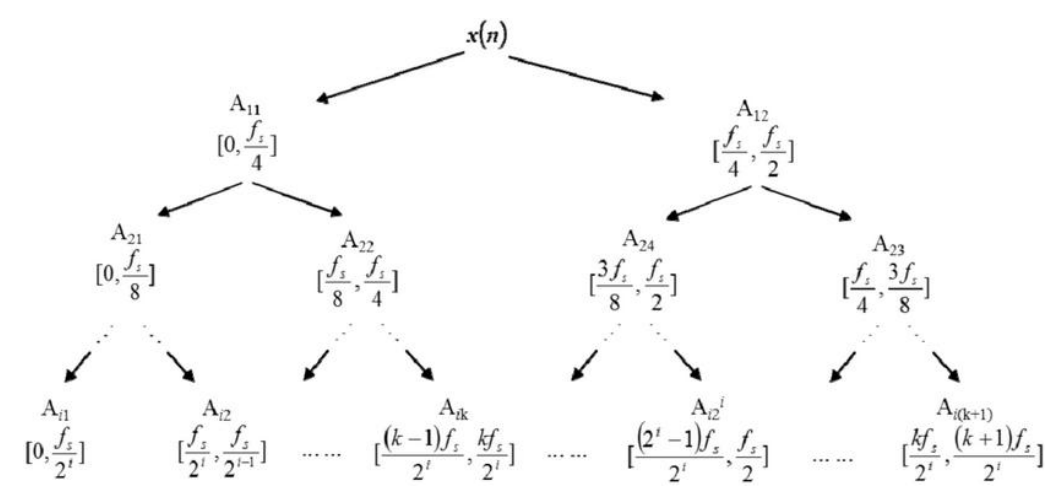

Figure 2. The Architecture of the Wavelet Packet Transform Model

Although the wavelet transform has been able to better meet the needs of the general, but for highly nonstationary, especially abundant high frequency components of the signal, wavelet transform is not effective enough. Because it in frequency domain is the division of the low frequency is more and more thin, high resolution and low frequency part and high frequency part of the resolution is not enough. In order to solve this basic problem, the wavelet packet transform is developed after wavelet transform of more general transformation: it is not only the low frequency decomposition of wavelet transforms and break down in the high frequency part is further subdivision. Accordingly, the related steps of the wavelet packet transform model can be reflected from the following equations.

$$
g_{j}^{n}(t)=\sum_{l} d_{l}^{j, n} u_{n}\left(2^{j} t-l\right)
$$




$$
\begin{aligned}
& \left\{\begin{array}{l}
d_{l}^{j, 2 n}=\sum_{k} a_{k-2 l} d^{j+1, n} k \\
d_{l}^{j+1,2 n}=\sum_{k} b_{k-2 l} d^{j+1, n} k
\end{array}\right. \\
& d_{l}^{j+1, n}=\sum_{k}\left[h_{l-2 k} d_{k}^{j, 2 n}+g_{l-2 k} d_{k}^{2 n-1}\right]
\end{aligned}
$$

In wavelet packets, the signal de-noising algorithm is thought as the basic frame of the wavelet, the only difference is the wavelet packet analysis provides a more complex and more flexible means of analysis, because of the wavelet packet analysis to a layer of low frequency part and high frequency part further subdivided at the same time that has more accurate local analysis ability. Multiresolution analysis is carried out on the low frequency part of image decomposition, and on the high frequency part of image cannot be considered, because in the high frequency band whose frequency resolution is poor and the wavelet packet transforms not only the low frequency part of image decomposition, but also to three high frequency part of image decomposition [7-8].

The Image De-blur Algorithm. Image restoration technology is concentrated in two main parts, parameter identification and filtering. Usually divided into two ideas, one is the primary parameter identification with the parallel structure of the reconstruction filter at the same time, the other is the advanced parameter identification, the reconstruction of degraded with the identified parameters do to recover the order of filter structure information, this article mainly adopts the second way of thinking. In fact, the two parts is most important parameter identification, if accurate parameter identification, the result is that always the available right, accordingly, the image model can be expressed as listed.

$$
h(\lambda, f)=\|H f-g\|^{2}+\lambda\|D f\|^{2}
$$

On the other hand, if fuzzy length of a pixel even smaller, so is sufficient to determine the image sharpening technology. Based on these premises, determined using band-pass filter for general image preprocessing, band-pass filter parameter selection is based on primary analysis of a large number of experimental results. Because of the relative motion of the camera and the target scene such as plane actually recorded information is part of the original target information, and the part is beyond the scope as flat as a meta information is lost, and in the exposure time, the greater the relative speed of imaging system and the target scene, blurred, the more information lost, namely the observations are zero background image and the filtering structure can be organized as follows.

$$
H_{\text {filter }}(u, v)=1-1 /\left(1+\left[\frac{D(u, v) W}{D^{2}(u, v)-D_{0}^{2}}\right]^{2 n}\right)
$$

Referred to in the introduction of algorithm includes two processes, this paper respectively on the restraint and elimination to the fuzzy and fuzzy noise spot removal. Compared with noise, image blur is statistical feature between the pixel and the constraint each other, so when removing the fuzzy can choose contain global features of wavelet redundant dictionary. Image spectrum contains "speckle structure" of the estimated parameters need easily masked by noise and high frequency structure, so we should remove the high frequency structure in determining the motion blur caused by the parallel stripe frequency spectrum filtering pretreatment should be first. In fact, depend on the length of the motion blur filter design. The optimization procedure can be reflected from equation 8 .

$$
\min \|y-H D \alpha\|_{2}^{2}+\lambda\|\alpha\|_{1}
$$




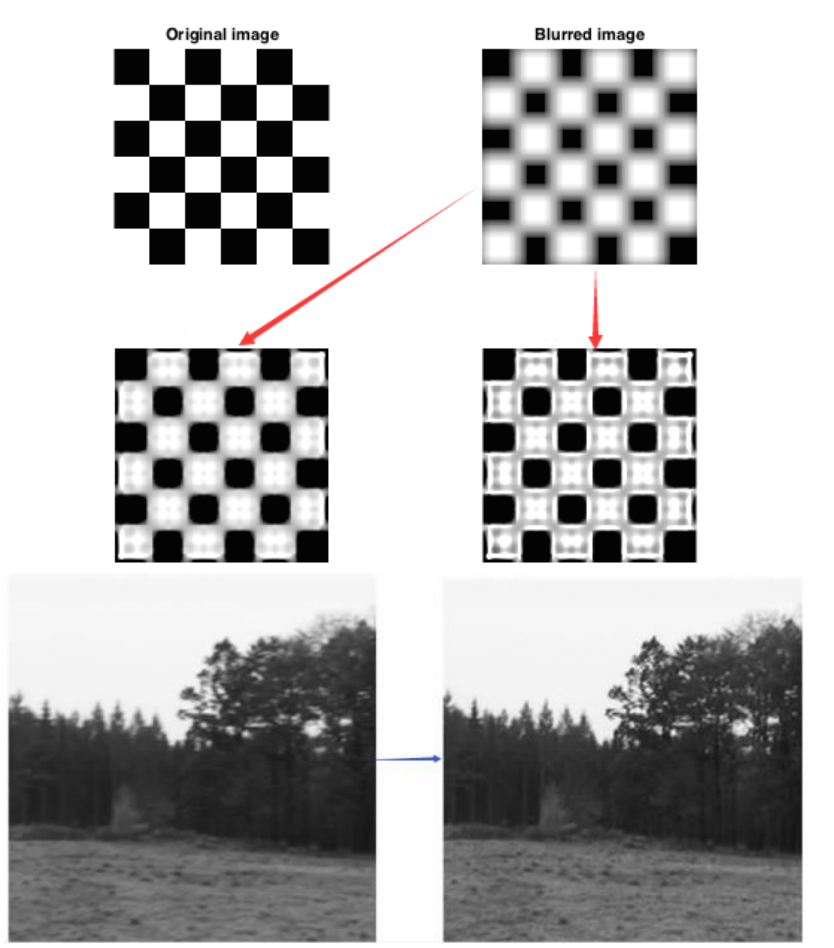

Figure 3. The Verification of the Image De-blur Algorithm

\section{Summary and Conclusion}

In this paper, we propose the novel image de-blur algorithm based on the wavelet packet transform model and the framework. Puts forward the multi-level image enhancement algorithm based on basic wavelet transform. The image wavelet decomposition levels in the first place, get the scale coefficient and the multiple levels of wavelet coefficients; Then the different levels of wavelet coefficients in the different enhancement algorithm for processing, and the coefficient of the scale of the image using multi-scale method for processing and finally wavelet coefficients and scale coefficient obtained by using the inverse transformation to get the enhanced image. This method makes the original images of different resolution by wavelet transform characteristics on the different scales are the details of the separation, recycle nonlinear transformation function component of the different scales of wavelet transform respectively and make the details of the original image of different resolution features have been enhanced. The experimental verification shows the effectiveness of the algorithm.

\section{References}

[1] Z. Hu and M. H. Yang: International Journal of Computer Vision, Vol.115 (2015) No.3, p.345.

[2] D. Rosenbaum and Y. Weiss: Annual Conference on Neural Information Processing System (Montreal, Canada, December 7-12, 2015). Vol. 3, p. 2683.

[3] Z. M. Deng, Y. X. Zhang, M. L. Tao and K. W. Yang: Advances in Multimedia, Vol. 2015 (2015), p. 285969.1 .

[4] S. H. Ye, C. Deng, J. Xu and X. B. Gao: IEEE International Conference on Acoustics, Speech and Signal Processing (Brisbane, Queensland, Australia, April 19-24, 2015). p. 1196.

[5] M. V. Afonso, J. A. Miguel and R. Sanches: IEEE Transactions on Image Processing, Vol. 24 (2015) No. 7, p. 2239.

[6] Y. J. Li, K. Lee and Y. Bresler: IEEE Transactions on Information Theory, Vol. 62 (2016) No. 7 , p. 4266. 
[7] Y. Chen, J. Wu and G. H. Yu: Statistics, Optimization \& Information Computing, Vol. 3 (2015) No. 1, p. 15.

[8] H. Yang, M. Zhu, Y. Niu, Y. J. Guan and Z. B. Zhang: 2015 IEEE Conference on Computer Vision and Pattern Recognition (Boston, MA, USA, June 7-12, 2015). p.705. 